\title{
ABSORPTION CROSS-SECTION AND DENSITY MEASUREMENT OF DYE VAPORS
}

\author{
J. SCHMIDT and A. PENZKOFER \\ Naturwissenschaftliche Fakultät II - Physik, Universität Regensburg, D-8400 Regensburg, FRG
}

Received 2 July 1987

\begin{abstract}
The absolute absorption cross-section spectrum of dimethyl-POPOP vapor is measured and compared with solution spectra. The dye vapor density versus temperature is determined by absorption coefficient measurement. Calibration is achieved by evaporation of a fixed amount of dye.
\end{abstract}

\section{Introduction}

In the vapor phase a solvent-free spectroscopic analysis of dye molecules is applicable [1-3]. For some dye vapors laser action was achieved [1-3]. The dye vapor pressure rises approximately exponentially with temperature. Reasonable vapor pressures ( $p$ around $100 \mathrm{~Pa}=1 \mathrm{mbar}$ ) are obtained for many non-ionic dyes in a temperature region between 200 and $400^{\circ} \mathrm{C}$ without considerable thermal decomposition of the dye molecules [1-3]. Ionic dyes have lower vapor pressures and are more difficult to transfer to the vapor phase without decomposition [4-6].

Most dye vapor absorption and emission spectra are presented in relative units, since the number density of dye molecules in the gas phase is seldom determined. In ref. [4] the vapor pressure of some dyes was measured with an isoteniscope (a glass spring pressure gauge) [7]. If all dye molecules in a closed cell are evaporated the vapor pressure is determined by the amount of inputted dye and the absolute absorption cross-section spectrum may be obtained [8-11]. The method of flow of molecules through a calibrated orifice and dye condensation at a cooled plate [12] was sometimes applied [9-12].

In this paper the saturated dye vapor density and dye vapor pressure of dimethyl-POPOP (1,4-di-[2-(4methyl-5-phenyloxazolyl)]benzene) is determined as a function of temperature by absorption measurements. Calibration is achieved by studying the temperature dependence of the absorption for a fixed amount of inputted dye. The absolute absorption cross-section spectra of dimethyl-POPOP are measured for various vapor temperatures. In some measurements buffer gas $(\mathrm{He})$ was added to the dye vapor. The dye vapor absorption cross-section spectra are compared with liquid solution spectra (solvents cylohexane, toluene, benzene, chloroform and methanol). Relevant dye-dye desorption and dye-stainless steel desorption parameters are deduced.

\section{Experimental}

The experimental arrangement is sketched in fig. 1. The vapor cell is made of stainless steel (inner length $5 \mathrm{~cm}$, inner diameter $1 \mathrm{~cm}$ ). At the bottom of the cylindrical cell a dye reservoir is flanged, and at the top of the cell a high-temperature valve (Nupro type SS-4H-TW) is welded. The vapor cell windows are made of saphire (thickness $5 \mathrm{~mm}$ ). The windows are sealed with gold O-rings. The dye reservoir is sealed 


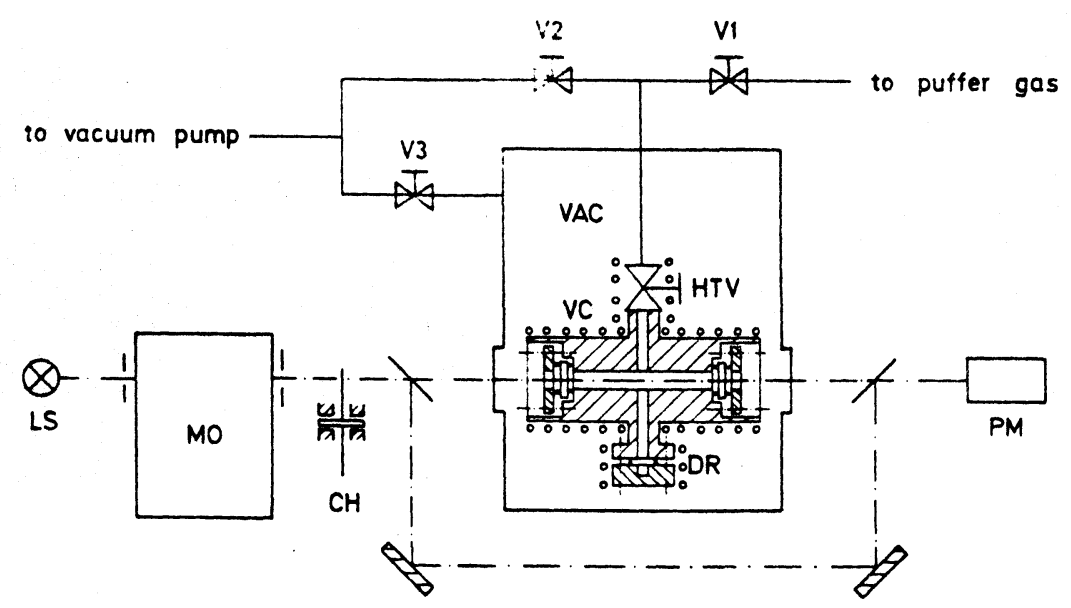

Fig. 1. Experimental setup for transmission measurements. LS, light source (tungsten lamp for $\lambda>350 \mathrm{~nm}$, deuterium lamp for $\lambda<350 \mathrm{~nm}$ ). MO, monochromator. $\mathrm{CH}$, light chopper. PM, photomultiplier. VAC, vacuum chamber. VC, vapor cell. DR, dye reservoir. HTV, high-temperature valve. V1-V3, valves. VC, DR and HTV are separately heated.

with $\mathrm{Ag}$ covered stainless steel gaskets. The main body of the vapor cell, the dye reservoir, and the valve are separately heated electrically (thermocoax cables of $6,12.5$, and $12.5 \Omega$, respectively, voltage $0-80 \mathrm{~V}$ ). Thermocouples $(\mathrm{Cr}-\mathrm{Ni}$-alumel) are used as temperature sensors. A home-made control unit regulates the temperatures.

The vapor cell is inserted into a vacuum chamber. The vapor cell itself is connected to a vacuum pump (cell evacuation down to about $10^{-2} \mathrm{~Pa}$ ) and to a buffer gas reservoir. The transmission measurements are carried out with a conventional UV-VIS spectrophotometer (Beckman model ACTA M4).

\section{Results}

The dye dimethyl-POPOP is investigated. Its structural formula is included in fig. 3. Three types of measurements are performed: (i) The absolute absorption cross section at the wavelength of peak $S_{0}-S_{1}$ absorption is determined by a procedure of transmission measurement at various temperatures for a fixed amount of dye in the vapor cell. (ii) The dye vapor absorption cross-section spectra at various temperatures are measured. The influence of the buffer gas He is studied. The vapor spectra are compared with liquid solution spectra. (iii) The saturated dye density in the vapor phase is measured versus temperature and the dye-dye desorption (sublimation or evaporation) energy is determined.

\subsection{Absolute absorption cross-section determination}

The absolute absorption cross section of the dye vapor is determined at the peak of the $S_{0}-S_{1}$ absorption band. A fixed amount of dye is inserted in the cell (degassed by preheating). The fixed dye input limits the dye density in the cell to a maximum density $N_{0}$. The dye reservoir is heated to temperatures $T_{\mathrm{R}}>T_{\mathrm{th}}$ where the saturated vapor density $N_{\mathrm{S}}$ is above $N_{0}$ so that no dye is left in the reservoir. The main cell and the valve are heated $15 \mathrm{~K}$ above the reservoir temperature (i.e. cell temperature $T=T_{\mathrm{R}}+15 \mathrm{~K}$ ). The following complication occurs: even in case of $T=T_{\mathrm{R}}+15 \mathrm{~K}>T_{\mathrm{th}}$ the vapor density in the cell $N$ is still less than $N_{0}$, since some amount of dye is adsorbed at the cell walls. 


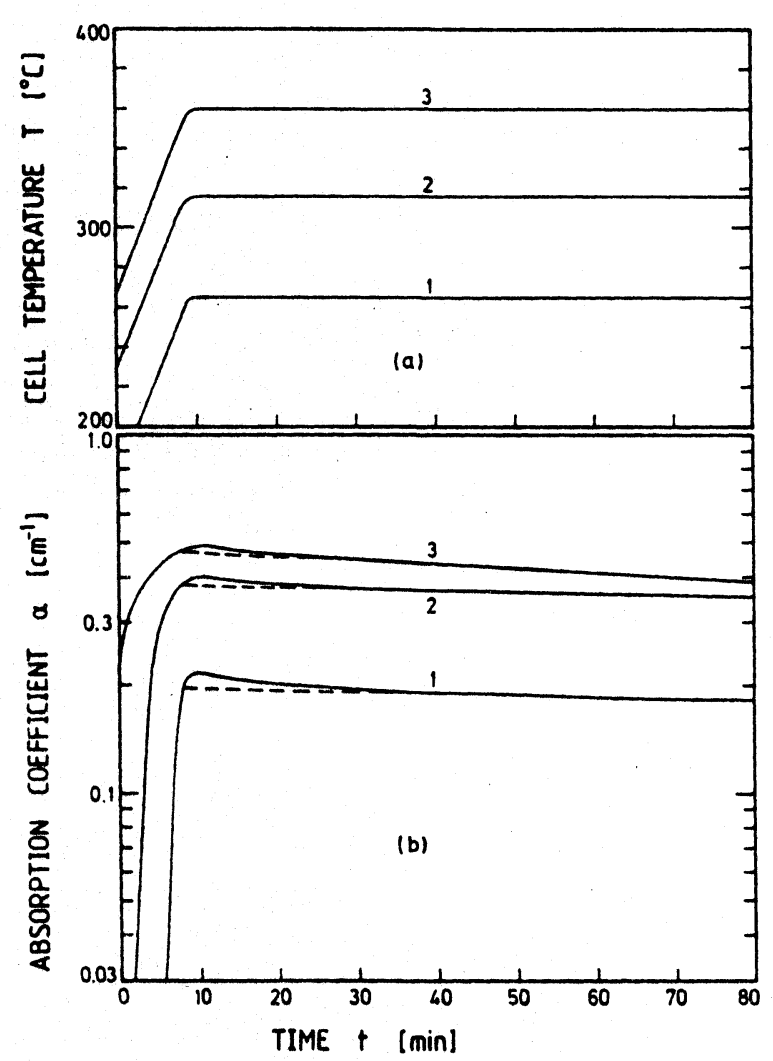

Fig. 2. Absorption coefficient measurement procedure for absorption cross-section determination. (a) Selected temporal temperature profiles. (b) Corresponding absorption coefficients versus time. Dashed curves are explained in text. Maximum dye number density $N_{0}=3.4 \times 10^{15} \mathrm{~cm}^{-3}$. Dye is dimethylPOPOP.

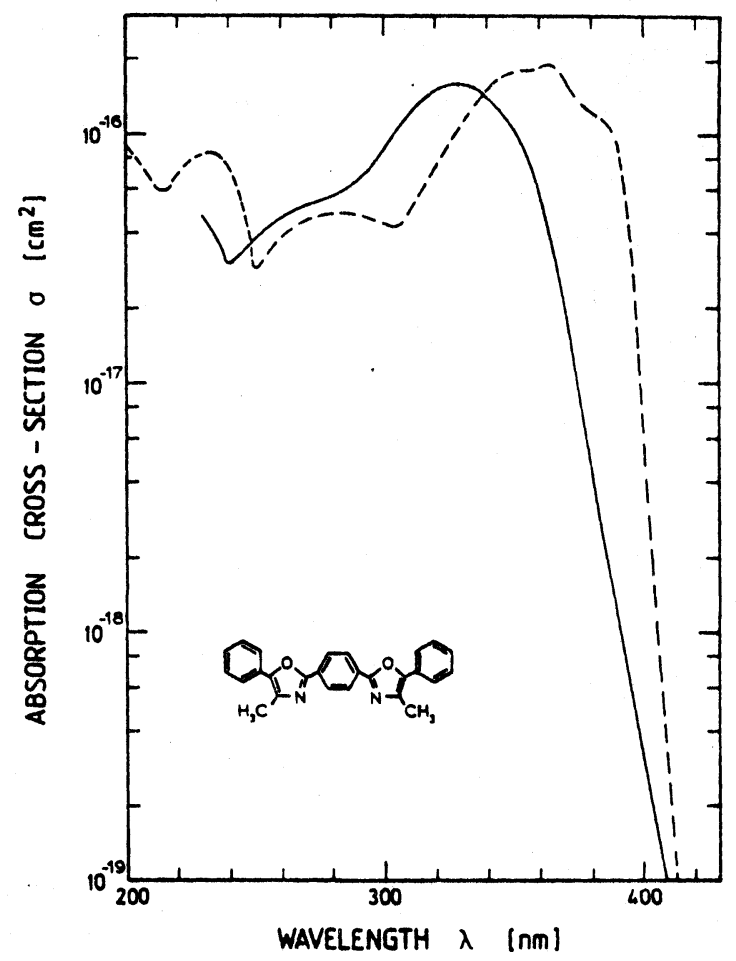

Fig. 3. Absorption cross-section spectrum of dimethyl-POPOP vapor at $T=260^{\circ} \mathrm{C}$ (solid curve) and of dimethyl-POPOP dissolved in cyclohexane (concentration $2 \times 10^{-4} \mathrm{~mol} / \mathrm{dm}^{3}$, $20^{\circ} \mathrm{C}$ ). Structural formula of dye is included.

With rising cell temperature $T$ above $T_{\text {th }}$ the vapor deposition at the cell walls reduces and the light transmission

$$
T_{\text {trans }}=\exp (-\alpha l)=\exp (-\sigma N l)
$$

approaches the limiting value $T_{\text {trans }, 0}=\exp \left(-\alpha_{0} l\right)=\exp \left(-\sigma N_{0} l\right)$.

In the experiments the transmission is measured at three cell temperatures $T_{1}, T_{2}, T_{3}$ with $T_{\mathrm{th}}<T_{1}<T_{2}$ $<T_{3}$. The corresponding absorption coefficients are $\alpha_{1}, \alpha_{2}$, and $\alpha_{3}$ with $\alpha_{1}<\alpha_{2}<\alpha_{3}<\alpha_{0}$. In fig. 2 the results are shown for three different runs at the cell temperatures $T_{1}=265^{\circ} \mathrm{C}, T_{2}=316^{\circ} \mathrm{C}$, and $T_{3}=360^{\circ} \mathrm{C}$. The vapor cell is heated up at a rate of $10 \mathrm{~K} / \mathrm{min}$ to the limiting temperatures $T_{1}, T_{2}$, and $T_{3}$. The temporal temperature profiles and the corresponding temporal dependences of the absorption coefficients are depicted in figs. $2 \mathrm{a}$ and $2 \mathrm{~b}$ respectively. Instead of three separate runs at different temceratures the analysis may also be carried out in a single run with successive rise of temperature from $\Gamma_{:}: 10 T_{2}$ and $T_{3}$ in due course after steady-state transmissions have settled in.

The limiting absorption coefficient $\alpha_{0}=\sigma N_{0}$ is calculated from the following considerations: 
A.: a vapor pressure $t$ the flux of dye molecules $n !\left(\mathrm{m}^{-2} \mathrm{~s}^{-1}\right)$ impinging the walls is [13]

$$
n_{\downarrow}=p /(2 \pi m i T):
$$

$m$ is the mass of a dye molecule, $k$ is the Boltzmann constant, and $T$ is the dye cell temperature. The surface density of adsorbed molecules $n_{\text {ad }}\left(\mathrm{m}^{-2}\right)$ is given by [14]

$$
n_{\text {ad }}(t)=n_{i} S_{\mathrm{wd}} \tau_{\mathrm{S}, \mathrm{wd}}\left[1-\exp \left(-t / \tau_{\mathrm{S}, \mathrm{wd}}\right)\right]
$$

$S_{\mathrm{wd}}$ is a sticking coefficient, $t$ is the time. Deposition starts at $t=0$. The steady-state density of adsorbed molecules is

$$
n_{\mathrm{ad}}=n_{\downarrow} S_{\mathrm{wd}} \tau_{\mathrm{S}, \mathrm{wd}} \text {. }
$$

The desorption time constant $\tau_{\mathrm{S}, \mathrm{wd}}$ is given by [14]

$$
\tau_{\mathrm{S}, \mathrm{wd}}=\left(1 / \nu_{\mathrm{wd}}\right) \exp \left(Q_{\mathrm{wd}} / k T\right) .
$$

$\nu_{w d}$ is the surface-vibrational frequency of the admolecules and $Q_{w d}$ is the wall-dye desorption energy. The vapor pressure $p$ is approximately related to the vapor density $N$ by the ideal gas equation

$$
p=N k T \text {. }
$$

The relations $n_{\text {ad }} A=\left(N_{0}-N\right) V=\left(\alpha_{0}-\alpha\right) V / \sigma$ ( $A$ is cell surface, $V$ is cell volume $)$ lead to the steady-state dependence

$$
\alpha_{0}-\alpha=\kappa_{\mathrm{wd}} \alpha T^{1 / 2} \exp \left(Q_{\mathrm{wd}} / k T\right),
$$

or

$$
\alpha_{0}=\alpha\left[1+\kappa_{\mathrm{wd}} T^{1 / 2} \exp \left(Q_{\mathrm{wd}} / k T\right)\right]
$$

with

$$
\kappa_{\mathrm{wd}}=(k / 2 \pi m)^{1 / 2}\left(S_{\mathrm{wd}} / \nu_{\mathrm{wd}}\right) A / V \approx(k / 2 \pi m)^{1 / 2}\left(S_{\mathrm{wd}} / \nu_{\mathrm{wd}}\right)(2 / r),
$$

$r$ is the inner cell radius ( $r=5 \mathrm{~mm}$ for our cell). Eq. (8) contains the three unknown quantities $\alpha_{0}, \kappa_{\mathrm{wd}}$ and $Q_{\text {wd }}$. They are determined by the known absorption coefficients $\alpha_{1}$ at $T_{1}, \alpha_{2}$ at $T_{2}$, and $\alpha_{3}$ at $T_{3}$. The final results are

$$
\begin{aligned}
& \alpha_{0}=\alpha_{3}\left(1+\frac{\left(\alpha_{3}-\alpha_{1}\right) T_{3}^{1 / 2} \exp \left(Q_{\mathrm{wd}} / k T_{3}\right)}{\alpha_{1} T_{1}^{1 / 2} \exp \left(Q_{\mathrm{wd}} / k T_{1}\right)-\alpha_{3} T_{3}^{1 / 2} \exp \left(Q_{\mathrm{wd}} / k T_{3}\right)}\right), \\
& \kappa_{\mathrm{wd}}=\frac{\alpha_{1}-\alpha_{2}}{\alpha_{2} T_{2} \exp \left(Q_{\mathrm{wd}} / k T_{2}\right)-\alpha_{1} T_{1} \exp \left(Q_{\mathrm{wd}} / k T_{1}\right)}
\end{aligned}
$$

and

$$
\begin{aligned}
\alpha_{2} / \alpha_{3} & =\left[T_{1}^{1 / 2} \exp \left(Q_{\mathrm{wd}} / k T_{1}\right)-T_{3}^{1 / 2} \exp \left(Q_{\mathrm{wd}} / k T_{3}\right)\right] \\
& \times\left\{T_{1}^{1 / 2} \exp \left(Q_{\mathrm{wd}} / k T_{1}\right)-T_{2}^{1 / 2} \exp \left(Q_{\mathrm{wd}} / k T_{2}\right)\right. \\
& \left.+\left(\alpha_{3} / \alpha_{1}\right)\left[T_{2}^{1 / 2} \exp \left(Q_{\mathrm{wd}} / k T_{2}\right)-T_{3}^{1 / 2} \exp \left(Q_{\mathrm{wd}} / k T_{3}\right)\right]\right\}^{-1}
\end{aligned}
$$

$Q_{w d}$ is obtained from the transcendental eq. (12). For the situation of fig. 2 (dimethyl-POPOP without buffer gas) the obtained wall-dye desorption energy is $Q_{\mathrm{wd}}=1.3 \times 10^{-19} \mathrm{~J}=0.8 \mathrm{eV}$. $\kappa_{\mathrm{wd}}$ has a value of 
$8 \times 10^{-11} \mathrm{~Pa} \mathrm{~K} \mathrm{~K}^{-1 / 2}$ giving $\nu_{\mathrm{wd}} / S_{\mathrm{wd}} \approx 1 \times 10^{13} \mathrm{~s}^{-1}\left(m=6.5 \times 10^{-25} \mathrm{~kg}\right.$, eq. (9)). Assuming a surfacevibrational frequency of $\nu_{\mathrm{wd}} \approx 10^{13} \mathrm{~s}^{-1}$ it results a sticking coefficient of $S_{\mathrm{wd}} \approx 1$ and a desorption time constant of $\tau_{\mathrm{S} \text {,wd }}=7 \times 10^{-7} \mathrm{~s}$ at $T=600 \mathrm{~K}$ (eq. (5)). The limiting absorption coefficient is $\alpha_{0}=0.54 \pm 0.03$ $\mathrm{cm}^{-1}$ for $N_{0}=3.4 \times 10^{15} \mathrm{~cm}^{-3}$ leading to $\sigma=(1.6 \pm 0.1) \times 10^{-16} \mathrm{~cm}^{2}$ at the used wavelength of $\lambda=330$ $\mathrm{nm}$.

In fig. $2 \mathrm{~b}$ the overshoots of the absorption coefficient $\Delta \alpha_{i}(i=1,2,3)$ over the dashed lines resemble the dynamics of the dye adsorption to the stainless steel walls (eq. (3)). It is mainly determined by the diffusion of dye molecules to the surfaces. The slopes of the dashed lines of fig. $2 b$ are thought to be due to thermal dye molecule decomposition and cell leakage.

The same absorption cross section $\sigma$ is found for dimethyl-POPOP vapor with $\mathrm{He}$ buffer gas (He pressure $=3.5 \times 10^{4} \mathrm{~Pa}$ at room temperature).

\subsection{Absorption cross-section spectra}

The absorption cross-section spectrum of dimethyl-POPOP vapor at $T=260^{\circ} \mathrm{C}$ without buffer gas is depicted in fig. 3. In the cell temperature range between 200 and $320^{\circ} \mathrm{C}$ the cross-section spectra are found to be independent of temperature. At higher temperature the short-wavelength absorption cross sections increase slightly, probably because of absorption of decomposed molecules. At $T=320^{\circ} \mathrm{C}$ a spectrum was also measured with $\mathrm{He}$ buffer gas $\left(3.5 \times 10^{4} \mathrm{~Pa}\right.$ at room temperature). Its spectrum is identical to the depicted spectrum in fig. 3 within our experimental accuracy.

The long-wavelength tail of the $S_{0}-S_{1}$ absorption band decreases exponentially according to $\sigma(\nu)=$ $\sigma\left(\nu_{0}\right) \exp \left[-h\left(\nu_{0}-\nu\right) / k T^{\prime}\right]$. For a cell temperature of $T=610 \mathrm{~K}$ a temperature of $T^{\prime} \approx 680 \mathrm{~K}$ is obtained. A long-wavelength exponential decrease of the apparent absorption coefficient $\sigma$ is expected by assuming thermal Boltzmann population of the vibrational states of the $S_{0}$ ground state $[15,16]$.

The absorption cross-section spectra of the dye vapors are compared with solution spectra of dimethyl-POPOP in methanol, cyclohexane, chloroform, toluene, and benzene. In fig. 3 the absorption cross-section spectrum of dimethyl-POPOP in cyclohexane is included. Spectroscopic data of the investigated solutions are collected in table 1.

Compared to the vapor spectra the solution spectra are shifted to longer wavelengths. A general formula for the shift of the pure electronic $S_{0}-S_{1}$ transition frequency, $\Delta \tilde{\nu}_{\text {abs }}=\tilde{\nu}_{\text {abs.vapor }}-\tilde{\nu}_{\text {abs.solution }}$, is derived in ref. [19]. It reads

$$
\Delta \tilde{\nu}_{\mathrm{abs}}=\left(J+\frac{\mu_{\mathrm{g}}^{2}-\mu_{\mathrm{e}}^{2}}{h c_{0} a^{3}}\right) \frac{n^{2}-1}{2 n^{2}+1}+\frac{2 \mu_{\mathrm{g}}\left(\mu_{\mathrm{g}}-\mu_{\mathrm{e}}\right)}{h c_{0} a^{3}}\left(\frac{D-1}{D+2}-\frac{n^{2}-1}{n^{2}+2}\right)+K \frac{\left(D-n^{2}\right)\left(2 D+n^{2}\right)}{D\left(n^{2}+2\right)^{2}} .
$$

Table 1

Spectroscopic data of dimethyl-POPOP

\begin{tabular}{|c|c|c|c|c|c|c|}
\hline \multirow[t]{2}{*}{ Parameter } & \multirow[t]{2}{*}{ Vapor } & \multicolumn{5}{|l|}{ Solution } \\
\hline & & methanol & cyclohexane & chloroform & toluene & benzene \\
\hline$\lambda_{\max }\left(S_{1}-S_{1}\right)(n m)$ & $330 \pm 1$ & $363 \pm 1$ & $364 \pm 1$ & $366 \pm 1$ & $368 \pm 1$ & $368 \pm 1$ \\
\hline$n\left(\lambda_{\max }\right)^{A 1}$ & 1.00 & 1.3437 & 1.4516 & 1.4703 & 1.5394 & 1.5502 \\
\hline$D^{h_{1}}$ & 1.00 & 33.64 & 2.023 & 4.8 & 2.379 & 2.284 \\
\hline$o\left(\lambda_{\max }\right)\left(\mathrm{cm}^{2}\right)$ & $1.6 \times 10^{-16}$ & $1.8 \times 10^{-16}$ & $1.9 \times 10^{-16}$ & $1.7 \times 10^{-16}$ & $1.75 \times 10^{-16}$ & $1.7 \times 10^{-16}$ \\
\hline $\int_{\mathrm{dh}} \sigma(\bar{i}) \mathrm{d} \bar{\nu}^{\mathrm{Cl}}(\mathrm{cm})$ & $9.5 \times 10^{-13}$ & $9.1 \times 10^{-13}$ & $9.5 \times 10^{-13}$ & $8.6 \times 10^{-13}$ & $8.7 \times 10^{-13}$ & $8.4 \times 10^{-13}$ \\
\hline$D_{\min }\left(\mathrm{cm}^{-1}\right)$ & 0 & 2755 & 2830 & 2981 & 3130 & 3130 \\
\hline
\end{tabular}


$I$ is a molecule-dependent constant, $\mu_{\mathrm{g}}$ and $\mu_{\mathrm{c}}$ are the permanent ground-state and excited-state dipole moments, respectively, $h$ is Planck's constant, $c_{0}$ is th ' vacuum light velocity, $a$ is the molecule radius, $n$ is the optical refractive index at the excitation frequency and $D$ is the static dielectric constant. $K$ is a constant that depends on solute and solvent parameters. Eq. (11) has to be modified if solute-solvent hycirogen bonds are formed or charge transfer occurs.

For nonpolar molecules in nonpolar solvents eq. (13) reduces to

$$
\Delta \bar{i}_{\text {abs }}=J\left(n^{2}-1\right) /\left(2 n^{2}+1\right) \text {. }
$$

The frequency shift is due to dispersion force interaction. The situation applies to dimethyl-POPOP in cyclohexane, toluene, and benzene.

For nonpolar molecules in polar solvents eq. (13) reduces to

$$
\Delta \tilde{\nu}_{\mathrm{abs}}=J\left(n^{2}-1\right) /\left(2 n^{2}+1\right)+K\left(D-n^{2}\right)\left(2 D+n^{2}\right) / D\left(n^{2}+2\right)^{2} .
$$

The second term describes the solvent Stark effect (solvent permanent dipoles induce field to solute and cause frequency shifts). This situation applies approximately to dimethyl-POPOP in methanol and chloroform.

In fig. 4 the frequency shift of the $S_{0}-S_{1}$ absorption peaks, $\Delta \tilde{\nu}_{\text {abs,p }}=\lambda_{\max }^{-1}$ (vapor) $-\lambda_{\max }^{-1}$ (solution). is plotted versus $\left(n^{2}-1\right) /\left(2 n^{2}+1\right)$ for the investigated solutions. The spectroscopic data are listed in table

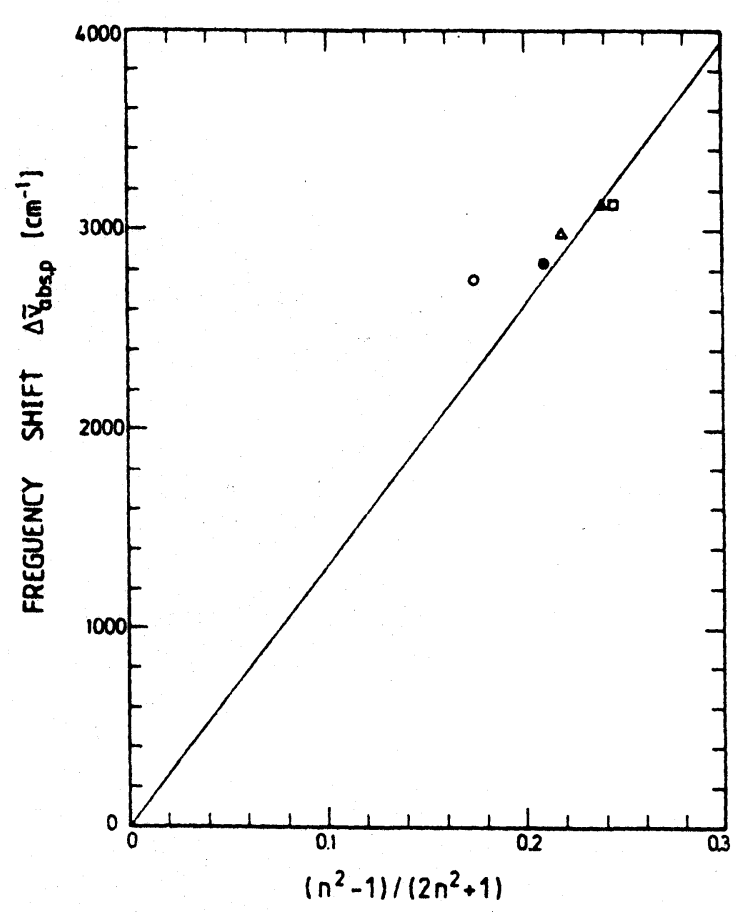

Fig. 4. Frequency shift, $\Delta \tilde{\nu}_{\text {aba, }}=\lambda_{\max }^{-1}$ (vapor) $-\lambda_{\max }^{-1}$ (solution), versus $\left(n^{2}-1\right) /\left(2 n^{2}+1\right)$ for dimethyl-POPOP dissolved in methanol $(O)$, cyclohexane $(\bullet)$, chloroform $(\Delta)$, toluene $(\Delta)$, and benzene ( $\square$ ). Used spectroscopic data are listed in table 1.

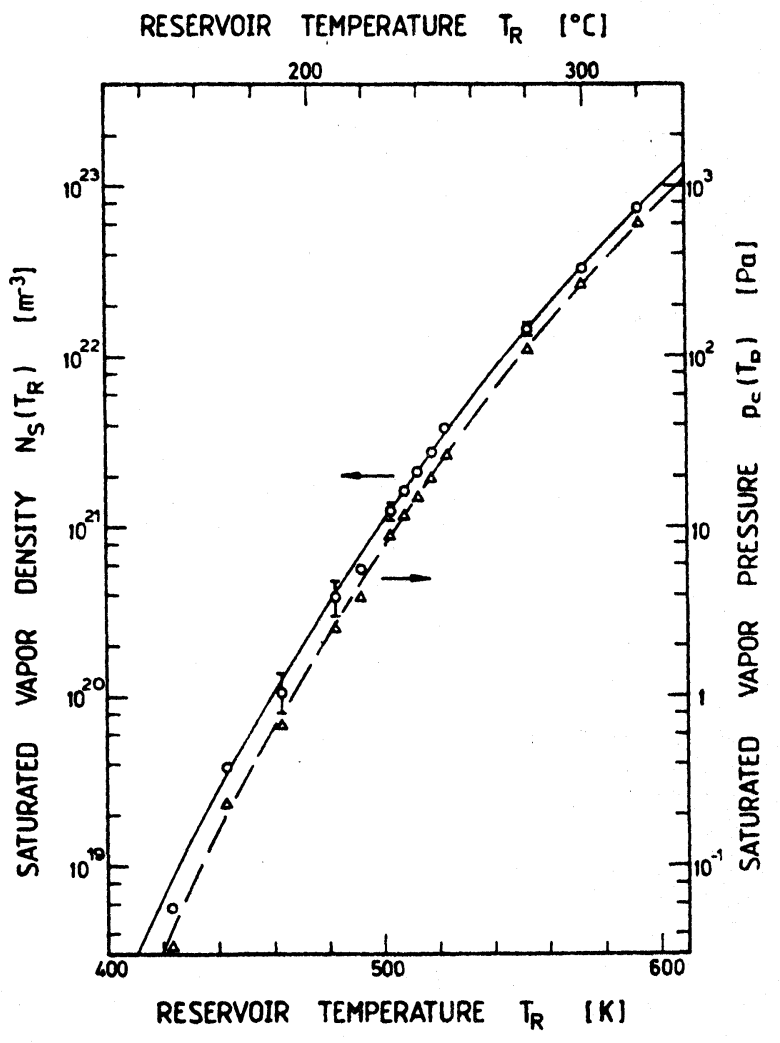

Fig. 5. Saturated vapor density (circles and solid curve) and saturated vapor pressure (triangles and dashed curve) versus reservoir temperature. Dye is dimethyl-POPOP. 
1. The frequency shift of the absorption peaks $\Delta_{a b s, p}$ is identical to the pure electronic transition frequency shift $\Delta \tilde{\nu}_{\text {abs }}$, if the Franck-Condon shift is the same in the vapor phase and in the liquid solutions. The data are reasonably well described by eq. (14) (cyclohexane, toluene, benzene) and eq. (15) (methanol, chloroform) with the parameters $J \approx 13100 \mathrm{~cm}^{-1}$ and $K \approx 100 \mathrm{~cm}^{-1}$.

The spectrally integrated $S_{0}-S_{1}$ absorption cross sections of the dye vapor and of the solutions are equal within our experimental accuracy (see table 1).

\subsection{Saturated vapor density and saturated vapor pressure}

The saturated vapor density as a function of temperature is determined with surplus dye in the reservoir. The vapor cell is heated up to a maximum temperature and is then cooled down in small temperature intervals. After each cooling step the temperature is held constant until the steady state transmission has been approached.

From the measured absorption coefficient $\alpha\left(\lambda, T_{\mathrm{R}}, T\right)$ and the known absorption cross section $\sigma(\lambda)$, the saturated vapor density $N_{\mathrm{S}}\left(T_{\mathrm{R}}\right)$ is obtained by

$$
N_{\mathrm{S}}\left(T_{\mathrm{R}}\right)=\left[\alpha\left(\lambda, T_{\mathrm{R}}, T\right) / \sigma(\lambda)\right] T / T_{\mathrm{R}} .
$$

$T_{\mathrm{R}}$ is the reservoir temperature and $T$ is the cell temperature. The factor $T / T_{\mathrm{R}}$ is derived from eq. (6) with constant pressure $p$.

A determination of the saturated vapor density by slowly heating up the vapor cell is not advantageous, because at low temperatures the equilibrium vapor pressure is very slowly approached. The dye adsorption to the vapor cell walls does not influence the measurements as long as there is condensed dye left in the reservoir.

The measured $N_{\mathrm{S}}\left(T_{\mathrm{R}}\right)$ data are presented in fig. 5 by circles. The triangles belong to the saturated vapor pressure $p_{\mathrm{S}}\left(T_{\mathrm{R}}\right)$ and are obtained by use of eq. (6).

The temperature dependence of the saturated vapor pressure may be derived by a similar procedure as applied to the determination of $\alpha_{0}$ (eqs. (2)-(6)). At equilibrium the rate of adsorbed dye molecules is equal to the rate of desorbed molecules. The density of adsorbed molecules, $n_{\mathrm{ad}}=n_{\downarrow} S_{\mathrm{dd}} \tau_{\mathrm{s}, \mathrm{dd}}$, remains constant and is equal to the surface density $n_{\text {surf }}\left(\mathrm{m}^{-2}\right)$ of dye molecules in the condensed phase, i.e.

$$
n_{\text {surf }}=n_{\downarrow} S_{\mathrm{dd}} \tau_{\mathrm{S} . \mathrm{dd}}=\left[p_{\mathrm{S}} /\left(2 \pi m k T_{\mathrm{R}}\right)^{1 / 2}\right]\left(S_{\mathrm{dd}} / \nu_{\mathrm{dd}}\right) \exp \left(Q_{\mathrm{dd}} / k T_{\mathrm{R}}\right)
$$

$\tau_{\mathrm{S} \text {.dd }}$ is the desorption time constant of dye molecules from the condensed dye, $S_{\mathrm{dd}}$ is a sticking coefficient of impinging dye molecules, $\nu_{\mathrm{dd}}$ is the surface-vibrational frequency of dye molecules at the surface of the condensed dye $Q_{\text {dd }}$ is the dye-dye desorption energy. It is equal to the sublimation energy below the melting point $\left(232^{\circ} \mathrm{C}\right.$ in case of dimethyl-POPOP) and to the evaporation energy above the melting point.

The saturated vapor pressure is obtained from eq. (17). It is

$$
p_{\mathrm{S}}\left(T_{\mathrm{R}}\right)=n_{\text {surf }}\left(2 \pi m k T_{\mathrm{R}}\right)^{1 / 2}\left(\nu_{\mathrm{dd}} / S_{\mathrm{dd}}\right) \exp \left(-Q_{\mathrm{dd}} / k T_{\mathrm{R}}\right)=\kappa_{\mathrm{dd}} T_{\mathrm{R}}^{1 / 2} \exp \left(-Q_{\mathrm{dd}} / k T_{\mathrm{R}}\right) .
$$

The saturated vapor density is obtained from eq. (18) by use of eq. (6):

$$
N_{\mathrm{S}}\left(T_{\mathrm{R}}\right)=n_{\text {surf }}\left(2 \pi m / k T_{\mathrm{R}}\right)^{1 / 2}\left(\nu_{\mathrm{dd}} / S_{\mathrm{dd}}\right) \exp \left(-Q_{\mathrm{dd}} / k T_{\mathrm{R}}\right)=\left(\kappa_{\mathrm{dd}} / k T_{\mathrm{R}}^{1 / 2}\right) \exp \left(-Q_{\mathrm{dd}} / k T_{\mathrm{R}}\right) .
$$

The calculated $p_{\mathrm{S}}$-curve (dashed) and $N_{\mathrm{S}}$-curve (solid) are depicted in fig. 5. The fitting parameters are $x_{\mathrm{dd}} \approx 2.5 \times 10^{11} \mathrm{~Pa} \mathrm{~K}{ }^{-1 / 2}$ and $Q_{\mathrm{dd}} \approx 1.9 \times 10^{-19} \mathrm{~J}=1.2 \mathrm{eV}$. Assuming a surface density of $2 \times 10^{18} \mathrm{~m}^{-2}$ one finds $\nu_{\mathrm{dd}} / S_{\mathrm{dd}}=1.7 \times 10^{16} \mathrm{~s}^{-1}\left(m=6.5 \times 10^{-25} \mathrm{~kg}\right)$. For a surface-vibrational frequency of $\nu_{\mathrm{dd}} \approx 10^{13}$ it results a sticking coefficient of $S_{\mathrm{dd}} \approx 6 \times 10^{-4}$. A desorption time constant of $\tau_{\mathrm{S} \text {,dd }} \approx 9 \times 10^{-4} \mathrm{~s}$ at $T=000 \mathrm{~K}$ is estimated (eq. (5)). The calculated saturated vapor pressure and vapor density curves follow the experimental points very well over a region of about five orders of magnitude. 
Table ?

Collisions in rure and buffered dye vapors. Dye: dimethyl-POPOF Buffer gas: helium of density $N_{\mathrm{g}}=8.65 \times 10^{24} \mathrm{~m} \cdot 1 p_{\mathrm{H}}=3.5 \times$ $10^{4}$ Pa: a: room temperature)

\begin{tabular}{llll}
\hline Cell temnerature & $\begin{array}{l}\text { Dye concentration } \\
\left.7,{ }^{\circ} \mathrm{C}\right)\end{array}$ & $\begin{array}{l}\text { Dye-dye collision time } \\
t_{\mathrm{c}, \mathrm{dd}}(\mathrm{s})\end{array}$ & $\begin{array}{l}\text { Dye-gas collision time } \\
l_{\text {c.gd }}(\mathrm{s})\end{array}$ \\
\hline $2 \vdots$ & $\left.2.2 \times 1 \mathrm{~m}^{-3}\right)$ & $1 \times 10^{-5}$ & $1.4 \times 10^{-10}$ \\
$\vdots 15$ & $3.3 \times 10^{22}$ & $6.5 \times 10^{-8}$ & $1.3 \times 10^{-10}$ \\
415 & $1.04 \times 10^{24}$ & $1.8 \times 10^{-9}$ & $1.2 \times 10^{-10}$ \\
\hline
\end{tabular}

The mean time between two collisions of evaporated dye molecules in the vapor cell is given by [13]

$$
t_{\mathrm{c}, \mathrm{dd}}=N / 2 Z_{\mathrm{dd}}=\frac{1}{2}\left[4 \pi a^{2}(4 k T / \pi m)^{1 / 2} N\right]^{-1} \text {. }
$$

$Z_{\mathrm{dd}}$ is the number of collisions per unit volume and per unit time, $a$ is the dye molecule radius $(a \approx 0.4$ $\mathrm{nm}), N$ is the density of dye molecules in the vapor phase. In table 2 dye-dye collision times are listed for three cell temperatures. Since the fluorescence lifetime of dimethyl-POPOP in the vapor phase is approximately $0.4 \mathrm{~ns}[20,21]$, dye-dye collisions in the excited state are negligible in the applied temperature range.

For dye vapors with buffer gas the time period between two collisions of buffer gas molecules with a dye molecule is given by [13]

$$
t_{\mathrm{c}, \mathrm{gd}}=N / Z_{\mathrm{gd}}=\left[\pi d^{2}(8 k T / \pi \mu)^{1 / 2} N_{\mathrm{g}}\right]^{-1}
$$

$Z_{\mathrm{gd}}$ is the number of collisions between buffer gas and dye molecules per unit volume and unit time, $d=a+a_{\mathrm{g}}$ is the sum of the molecule radii of a dye molecule $(a)$ and a buffer gas molecule $\left(a_{\mathrm{g}}\right)$, $\mu=m_{\mathrm{g}} m /\left(m+m_{\mathrm{g}}\right)$ is the effective mass of a dye $(m)$-buffer gas $\left(m_{\mathrm{g}}\right)$ collision pair. For dimethyl-POPOP in the He buffer gas the parameters are $d \approx 0.4 \mathrm{~nm}$ and $\mu \approx m_{\mathrm{g}}=6.6 \times 10^{-27} \mathrm{~kg}$. Some calculated collision times for our experimental situation are listed in table 2. The dye-gas collision time is about a factor of three shorter than the fluorescence lifetime of dimethyl-POPOP, i.e. about three He atoms hit a dimethyl-POPOP molecule within its fluorescence lifetime.

\section{Conclusions}

A method is described to determine the absolute absorption cross-section spectrum of dyes in the vapor phase and to measure the saturated vapor pressure and vapor density. The technique is applied to dimethyl-POPOP. It may be used to the absorption cross section and vapor pressure measurement of any dye. The accurate knowledge of the vapor density and of the absorption spectrum is the prerequisite in the spectroscopic analysis of dye vapors and in the investigation of their laser applicability.

\section{Acknowledgement}

The authors thank Th. Ascherl for technical assistance and G. Gössl and E. Niedermeyer for developing the temperature control unit. They thank the Deutsche Forschungsgemeinschaft for financial support. 


\section{References}

[1] F.P. Schäfer, ed., Topics in applied physics, Vol. 1. Dye lasers, 2nd Ed. (Springer, Berlin, 1977).

[2] N.A. Borisevich and V.A. Tolkatchev, Soviet Phys. Usp. 25 (1982) 865.

[3] Yu.Yu. Stoilov, Appl. Phys. B 33 (1984) 63.

[4] B. Steyer and F.P. Schäfer, Appl. Phys. 7 (1975) 113.

[5] A.T. Vartanyan, Zh. Fiz. Khim. 30 (1956) 1028.

[6] M.M. Asimov, E.V. Portnov and A.N. Rubinov, Opt. Spectry. 60 (1986) 799.

[7] H. Kienitz, in: Houben-Weyl: Methoden der organischen Chemie, Vol. 3, Part 1, ed. E. Müller (Thieme, Stuttgart, 1955 ) p. 255.

[8] J.P. Maier, A. Seilmeier, A. Laubereau and W. Kaiser, Chem. Phys. Letters 46 (1977) 527.

[9] G.A. Abakumov, B.I. Polyakov and A.P. Simonov, Opt. Spectry. 45 (1978) 752.

[10] G.A. Abakumov, Yu.M. Anisimov, B.I. Polyakov and A.P. Simonov, Appl. Phys. 23 (1980) 83.

[11] G.A. Abakumov, S.A. Drobakha, A.V. Khimanov, A.V. Ostrovskii, B.I. Polyakov and A.P. Simonov, Soviet J. Quantum Electron. 16 (1986) 1566.

[12] J.D. Kelley and F.O. Rice, J. Phys. Chem. 68 (1964) 3794.

[13] P.W. Atkins, Physical chemistry, 2nd Ed. (Oxford Univ. Press, Oxford, 1982) p. 874.

[14] L. Eckertova, Physics of thin films, 2nd Ed. (Plenum Press, New York, 1986) p. 99.

[15] W. Blau, W. Dankesreiter and A. Penzkofer, Chem. Phys. 85 (1984) 473.

[16] A. Penzkofer and P. Sperber, Chem. Phys. 88 (1984) 309.

[17] K.H. Hellwege and Hellwege, eds., Landolt-Börnstein, 6th Ed.. Vol. 2, Part 8, (Springer, Berlin, 1962 ).

[18] D.E. Gray, ed., American Institute of Physics handbook, 3rd Ed. (McGraw-Hill, New York, 1972).

[19] M. Mataga and T. Kubota, Molecular interactions and electronic spectra (Dekker, New York, 1970) ch. 8.

[20] P.F. Liao. P.W. Smith and P.J. Maloney, Opt. Commun. 17 (1976) 219.

[21] T.F. Raichenok, J. Appl. Spectry. 41 (1984) 1217. 